\title{
Toxicity of bisphenol a on humans: a review
}

\author{
S. Preethi, K. Sandhya, D. Esther Lebonah, Ch. Venkata Prasad, B. Sreedevi, \\ K. Chandrasekhar, J. Pramoda Kumari* \\ Department of Microbiology, S.V. University, Tirupati, Andhra Pradesh, India \\ *E-mail address: prmodkumarij@gmail.com
}

\begin{abstract}
Bisphenol A (BPA) is a plastic ingredient produced in large quantities for use primarily in the production of polycarbonate plastics and epoxy resins. The present review focused on different mechanisms of BPA on human health in enzymatic, androgenic, neurological, liver and reproductive systems. The review explained the influence of BPA on different stages of human life likely in foetal stage, children and adults stages. The review also concentrated on how to handle BPA, treatment, preventive measures against BPA exposure.
\end{abstract}

Keywords: Bisphenol A; plastic ingredient; Endocrine Disruptor Compounds; Neurological effects; fetal studies; liver studies; reproductive system effect and adipose tissue

\section{INTRODUCTION}

\section{Sources of Bisphenol A:}

Bisphenol A (BPA) is one of the most highly produced industrial chemicals globally. It is used primarily for the manufacture of polycarbonate plastic and epoxy resins and such as used in a wide variety of consumer products ${ }^{1}$. BPA is a small $(228 \mathrm{Da})$ molecule which is used as a monomer in polymerization reaction to produce polycarbonate plastics. It exists at room temperature as a white solid and has a mild "phenolic" or hospital odor.

Over 2 billion pounds of BPA are used in the production of epoxy resins and polycarbonate plastics every year, these plastic products are often used in food and drink packaging creating a direct ingestion exposure pathway. The resins are used as lacquers for metal cans, bottle tops, and ground water pipes.

The manufacturing process of BPA involves the combination and condensation of one part of acetone with two parts of phenol (in the presence of a catalyst and promoter) under conditions of high temperatures and low $\mathrm{pH}$, followed by purification of the product ${ }^{2}$.

It is generally believed that consumer exposure to BPA occurs primarily via food in contact with BPA-containing materials, such as polycarbonate baby bottles, table ware and food containers as well as food and beverage cans lined with epoxy resins. Recently, it has also been shown that BPA can be transferred to the skin from certain types of thermal printing paper, such as some types of cashier's receipts, insignificant amounts ${ }^{3}$.

EDCs (Endocrine Disruptor Compounds) are compounds in the environment that imitate, block, alter, or otherwise modify endogenous hormonal activity. Alterations of hormone levels, receptors, or actions in the brain through exposure to EDCs are not only disruptive to the structure and function of the brain itself but also to the regulation of hormones downstream from the brain. Endocrine disruptors exert uncharacteristic exposure- 
effect relationships, because low doses often exert more potent effects than higher doses (NTP, 2001) BPA is a ubiquitous synthetic chemical in the environment and is present in nearly all human serum samples from developed countries ${ }^{4}$. BPA has been measured in human serum, urine, amniotic fluid, placental tissue and umbilical cord blood.

\section{Physical/Chemical Properties:}

BPA is a white to tan flake or powder with a mild odor. It is not volatile. Other properties include:

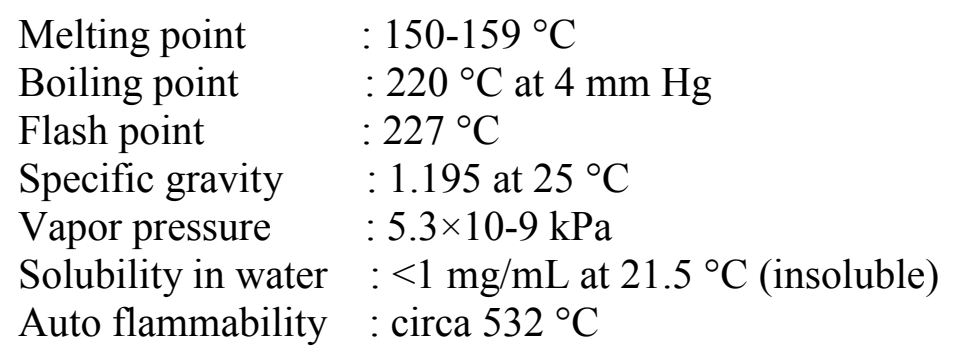

\section{Release of BPA from polycarbonate:}

BPA can leach from polycarbonate into liquid foods by two different processes:

1. After the manufacturing process of polycarbonate, the residual BPA present in it get hydrolysed (i.e) catalyzed by hydroxide $(-\mathrm{OH})$ in contact with aqueous food and simulants.

2. Diffusion is the only relevant process in case of dry foods. Release of BPA from polycarbonate containers into food depends on the contact time, temperature, and type of food.

3. Food simulants are often used in BPA release studies to represent the different types of food e.g. $50 \%$ of ethanol in water is the food simulant for milk, and $3 \%$ of acetic acid in water is the simulant for fruit juice.

\section{Human exposure to BPA:}

Individuals working in the manufacture of BPA containing products can be exposed to BPA at the workplace. More specifically, exposure to BPA can arise under conditions where residual monomer in the polymer migrates into food and beverages, or where the polymer itself hydrolyses, thereby releasing BPA. Other, relatively minor sources of consumer exposure to BPA are dental fissure sealants, epoxy-based surface coatings, adhesives, printing inks and thermal paper.

BPA may be the bonafide endocrine disruptor that adversely affects metabolic homeostasis. The following figure presents a model which incorporates input by environmental factors to the combined effects of diet, exercise and genetics on the development and manifestation of the metabolic syndrome

From a toxic kinetic viewpoint, it was found that BPA has a short half-life in the body and is thus considered a relatively less cumulative chemical ${ }^{5}$ and most of the dose absorbed as BPA is glucuronidated in rat liver and intestine following oral exposure ${ }^{6}$. Due to the short half-life of BPA, this chemical is excreted rapidly from the body by forming BPA glucuronidates or BPA sulfates during the metabolic process ${ }^{7,8}$. 


\section{MECHANISM OF BISPHENOL ON HUMAN HEALTH}

\section{A) Receptor-mediated mechanisms:}

The first characterized mechanism of action for endocrine disruptors was the ability to act directly as ligands for steroid hormone nuclear receptors (NRs), in particular estrogen, androgen, and thyroid hormone receptors. When a compound is bound to a receptor, it can have various effects. A substance that binds to a receptor and triggers a response is called an agonist. Agonists often mimic the action of the naturally occurring ligand, however, often with different potencies. In contrast to an agonist, an antagonist blocks the action of the agonist or natural ligand. Selective receptor modulators (SRMs) are receptor ligands that exhibit agonistic or antagonistic ability in a cell- and tissue dependent manner.

\section{B) Non-receptor-mediated mechanisms Interference with steroid genic enzymes and hormone transport:}

The ability of xenobiotics to disrupt steroidogenesis and the mechanisms by which these compounds interfere with the function of steroidogenic enzymes are very complex. The enzyme that has received the most attention with regard to endocrine disruptors is aromatase (CYP19) that converts androgens to estrogens. The cytochrome P450 enzymes responsible for the highly specific reactions in the steroid biosynthesis pathway are some of the molecular targets of interest, given their key role in the formation of various highly potent endogenous steroid hormones.

BPA at low nano molar concentrations suppressed adiponectin release from human adipose tissue explants, as well as from isolated mature adipocytes ${ }^{9}$. Adiponectin increases insulin sensitivity and reduces tissue inflammation; therefore any xenobiotic chemical that has the ability to suppress adiponectin release could lead to insulin resistance and increased susceptibility to obesity-related diseases ${ }^{9}$.

Several in vitro studies show in a human prostate carcinoma cell line bisphenol A can act as an androgen receptor antagonist and is reportedly mitogenic through interactions with a mutant tumor-derived form of the androgen receptor. In vitro studies, it is observed that Bisphenol A also interacts with thyroid hormone receptors (TRs) and reported to either inhibit TR-mediated transcription or inhibit the actions of triiodothyronine (T3) or its binding to TRs, or stimulate cell proliferation in a thyroid hormone responsive cell line. One in vivo study suggests that bisphenolA acts as a selective TR $\beta$ antagonist.

Bisphenol A may also inhibit activity of aromatase, the enzyme that converts testosterone to estradiol.

\section{C) BPA as Androgen receptor antagonist:}

The androgen receptor (AR) is the major regulatory element of androgen cell signaling and is essential for male reproductive function and development, including spermatogenesis. Several assays have shown that BPA acts as an androgen receptor antagonist ${ }^{10-12}$.

Aromatase is found in a number of tissues including the brain, testicular Leydig cells and adipose tissue. The aromatase enzyme is a key player in steroid synthesis as it catalyzes the irreversible conversion of androgens into estrogens.

BPA reduces aromatase enzyme activity by acting on two steroidogenic enzymes ${ }^{12}$, decreasing testosterone and E2 synthesis. 


\section{D) BPA as Polychlorinated Biphenyls (PCBs):}

PCBs are promiscuous compounds that may also act on neurotransmitter receptors, with strongest evidence for the dopaminergic system and hormonal targets that are not always predictable.

BPA can bind to both nuclear estrogen receptors alpha and beta (ER $\alpha$ and ER $\beta$ ) to induce estrogenic signals that act as transcriptional transactivators to regulate estrogenresponsive gene expression

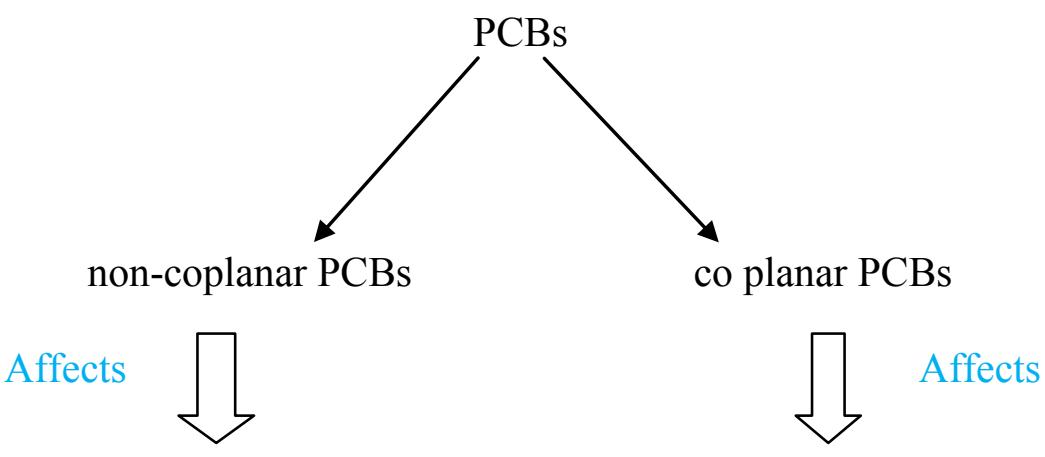

(dopamine, serotonin, and acetylcholine) (thyroid hormone, glucocorticoids)

Fig. 1. Effect of Polychlorinated Biphenyls on human body.

\section{1. Changes in humans with the influence of BPA}

\section{a) Neurological effects:}

The effects of BPA on the brain and behavior are assumed to be attributed to its estrogen receptor(ER)-mediated action, but it is not clear how its low potency could account for the strong effects that are observed in many tissues after exposure to relatively low doses

$\mathrm{Xu}$ et al., explained that the cultured hippocampal neurons, BPA exposure (10$1000 \mathrm{nmol} / 1$ for 30 minutes) rapidly promotes dynamic changes in dendritic morphology through the ER-mediated pathway by concomitant phosphorylation of $N$-methyl-D-aspartate (NMDA) receptor subunit NR2B.

There is also evidence that changes in gene expression in utero persist into adulthood ${ }^{13}$ and hence possibly involve epigenetic mechanism.

Ishido \& Suzuki (2010) found a dose-dependent (0-100 $\mu \mathrm{mol} / \mathrm{l})$ inhibitory effect of BPA on neural stem cell migration and proliferation.

\section{b) In vitro studies:}

In in-vitro studies, BPA-induced activation of protein kinase pathways (e.g. via the extracellular signal-regulated kinase $[E R K]$ ) has been associated with neurotoxicity (cell death) in PC12 cells, primary cortical cultures and hippocampal HT-22cells ${ }^{14,15}$. Using a neurosphere assay in vitro (isolated from GD16 rat mesencephalon),

\section{c) Hormonal effects:}

It is now well documented that numerous environmental chemicals can interfere with complex endocrine signaling pathways and cause adverse schemical effects: an Endocrine Society scientific statement. 
It is not surprising that PCBs alter hormonal levels and they are known to affect almost every aspect of the endocrine system. Exposure to PCBs during development alters adult reproductive and other sexually dimorphic behaviors.

Perinatal exposure to BPA has been associated with changes (usually a decrease) in brain expression levels of $\mathrm{TH}$, the rate-limiting enzyme for DA synthesis For example, neonatal exposure to A1254 reduces sexual receptivity (lordosis quotient), whereas A1221 has no effect. However, when treatment includes prenatal and postnatal exposure, A1254 decreases sexual motivation and A1221 decreases sexual receptivity.

Under the hypothesis that BPA action is primarily due to estrogenic activity, consideration must be given to species in interpretation of sexually dimorphic behaviors with regard to potential human effects, as rodent hormonal sexual differentiation is primarily controlled by estrogens, whereas similar pathways appear to be regulated by and particularly those that are estrogen sensitive

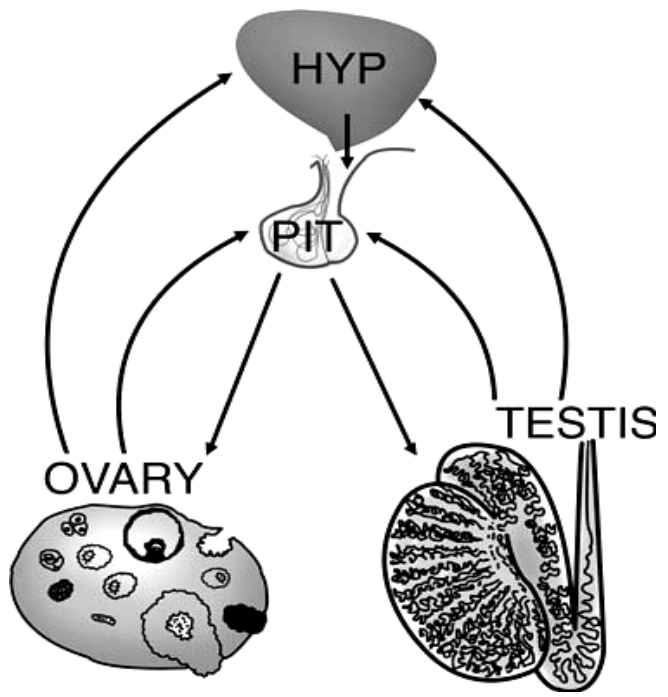

Fig. 2. Effect of BISPHENOL A on Glands and reproductive system.

\section{d) Effect on adipose tissue:}

One of the new emerging effects or mode of action for EDCs is that exposure to EDCs may play a role in obesity. Altered parameters of adipocyte biology that result in depot remodeling or gain of adipose mass leading to obesity would be a good confirmation of a link between EDCs and obesity.

However, to confirm the hypothesis about EDCs and their effect on the development of obesity, an appropriate molecular target affecting regulation of adipose physiology must be found. Using breast, subcutaneous and visceral adipose tissue explants as well as isolated mature adipocytes from over 20 patients, we reported that BPA at 1 and $10 \mathrm{nM}$ concentrations inhibits adiponectin release ${ }^{9}$. 


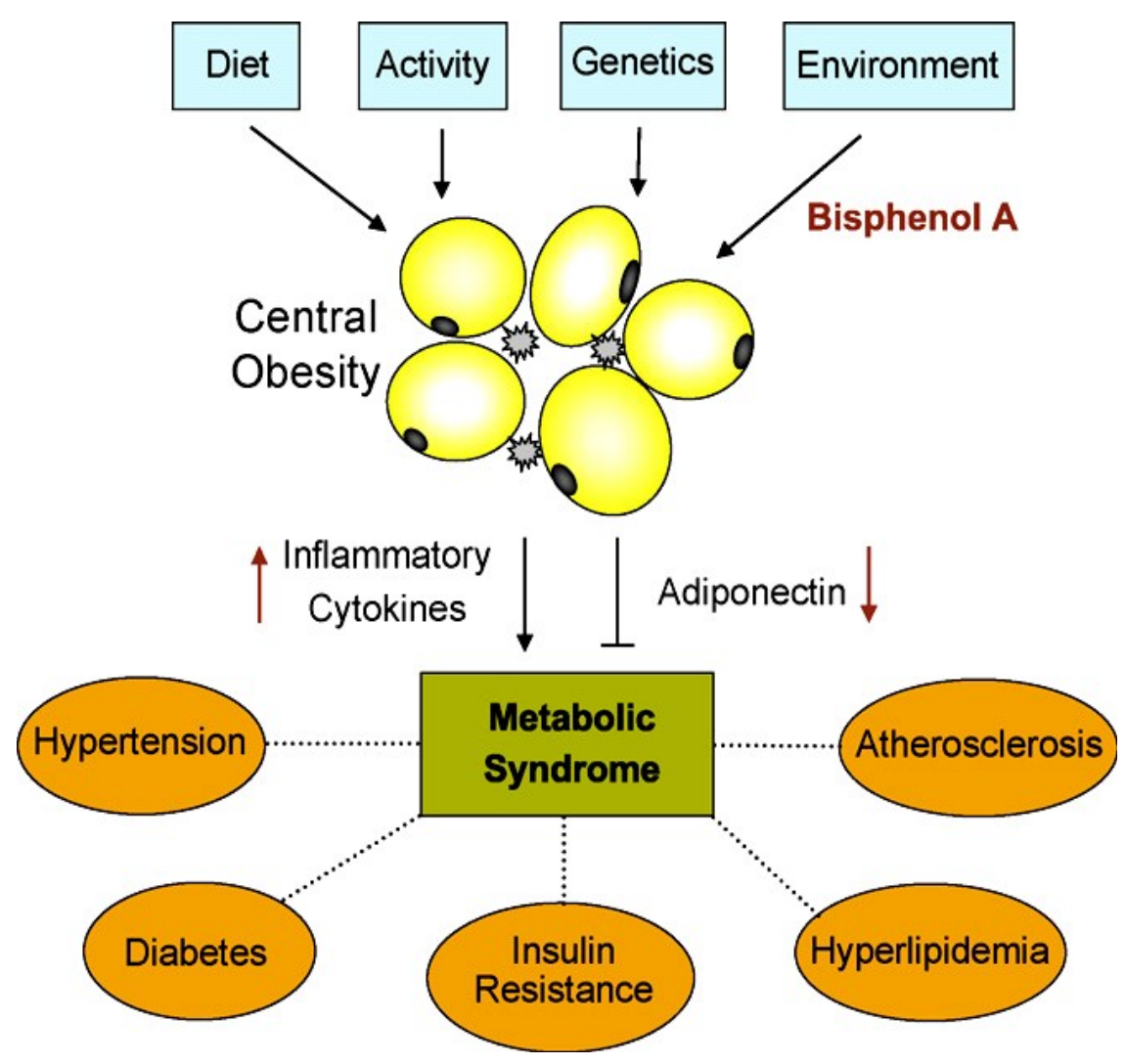

Fig. 3. A model depicting an integrated view of the various factors that affect the obesity-related metabolic syndrome.

\section{e) Effect on liver:}

The metabolic effects of BPA are critically important to recognize the full spectrum of its actions on human fat. The repeated administration of BPA has toxic effects on the liver in mice and it has been observed in previous studies with a lowest-observed-adverse-effect level (LOAEL) of $120 \mathrm{mg} / \mathrm{kg} \mathrm{bw/day,} \mathrm{suggesting} \mathrm{that} \mathrm{liver} \mathrm{toxicity} \mathrm{is} \mathrm{at} \mathrm{least} \mathrm{as} \mathrm{sensitive} \mathrm{an}$ endpoint for BPA as reproductive and developmental effects. BPA accumulation in rat adipose tissue was low in one study ${ }^{5}$, but high in another ${ }^{16}$.

\section{f) Effect on reproductive system:}

The reproductive toxicity studies of bisphenol A include:

1. Assessment of fertility

2. Sperm counts

3. Estrous cycling

4. Growth or cellular damage in reproductive tissues.

Reproductive health ultimately depends on the proper organization and function of the hypothalamic-pituitary-gonadal (HPG) axis, a neuroendocrine system involving the hypothalamus; the pituitary gland, lying just beneath the brain; and the gonads (ovaries in females, testes in males). BPA exposure can disrupt pubertal timing and compromise the capacity to maintain a regular ovulatory cycle in rodents and these defects result from the abnormal organization of the hypothalamic pituitary-gonadal axis, the crucial neuroendocrine pathway that regulates reproductive function. BPA has also been found to induce apoptosis 
and cell arrest in cultured ovarian granulosa cells, suggesting that BPA may also impact the adult ovary.

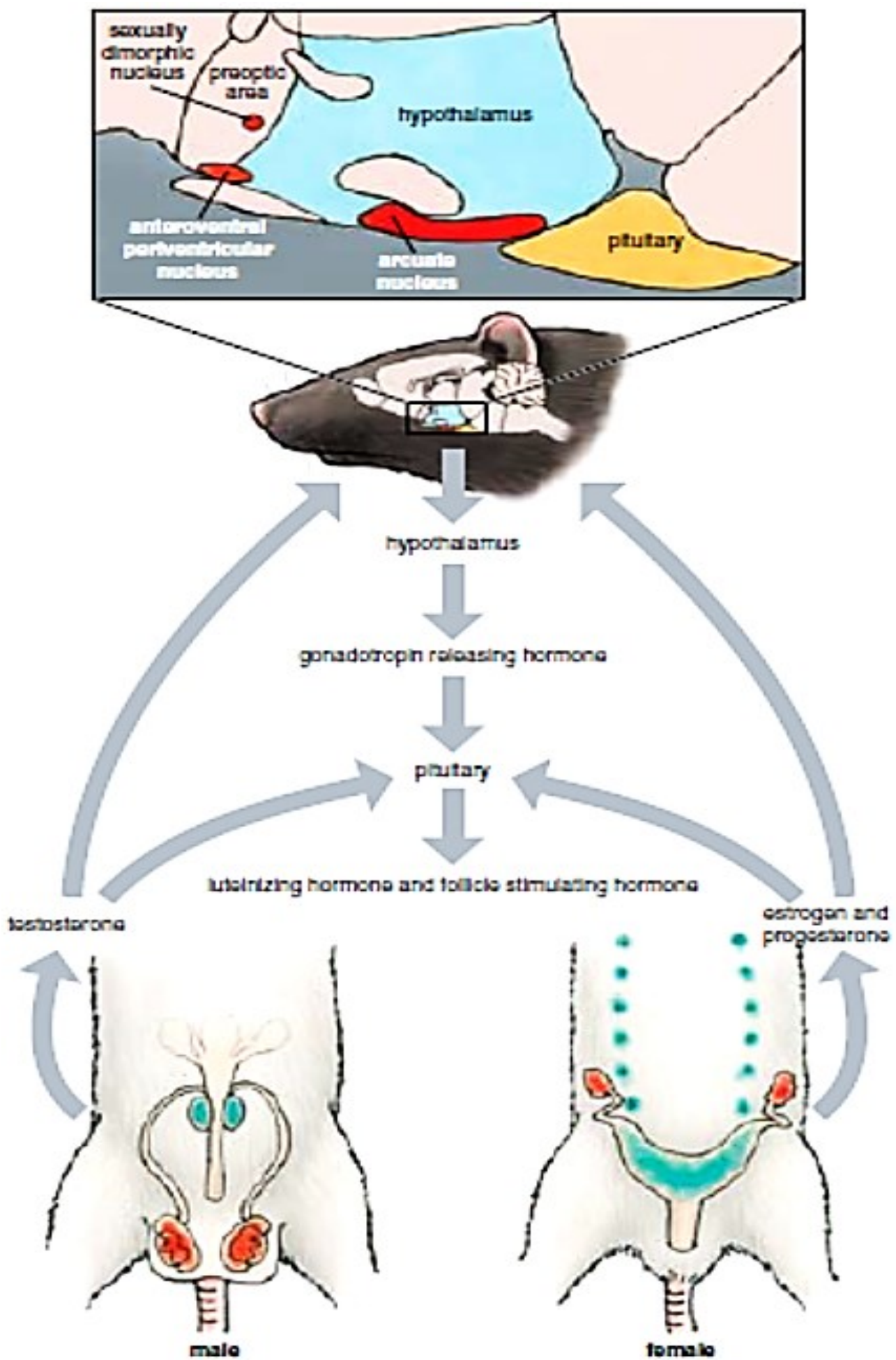

Fig. 4. Bisphenol A effects on reproductive system.

Specifically, exposure to doses of BPA relevant to normal human exposure during fetal development have been shown to 
1. Advance puberty

2. Increase prostatic growth

3. Alter pubertal mammary gland development

4. Permanently change the morphology and functionality of the female reproductive tract and ovaries in mice.

5. Compromise sexual differentiation in the brain.

\section{2. Metabolic changes in humans with the influence of BPA:}

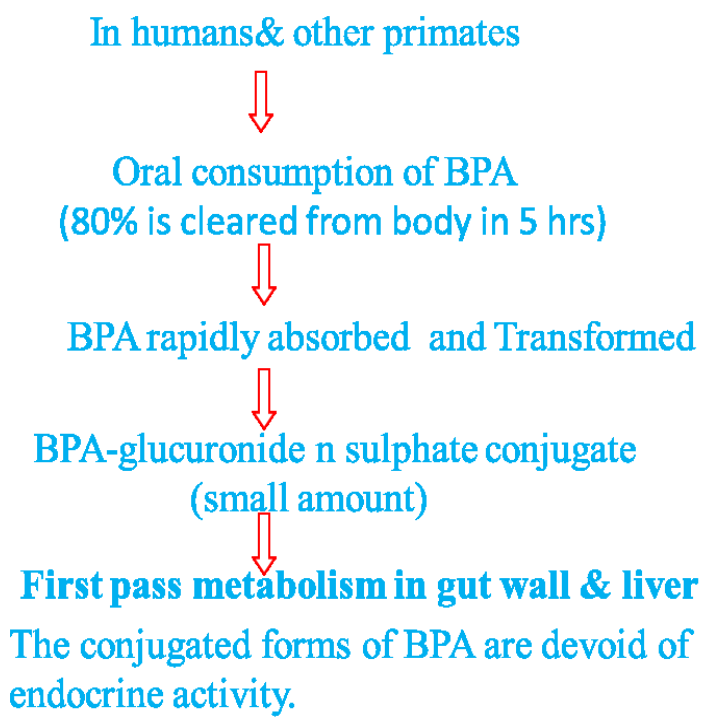

\author{
In rodents<smiles>C1=[Al]=C1</smiles> \\ Oral administration of BPA \\ $\sqrt{ }$ \\ Highly bound to plasma protein<smiles>[CH]1C=C1</smiles> \\ Excreted in the bile \\ Cleaved again to free BPA \\ Reabsorption from \\ Intestine into blood stream<smiles>[C]1=C=C1</smiles> \\ Before BPA is excreted \\ Entero heptic process occur several times
}

Fig. 5. Diagrammatic representation of metabolic changes in humans with the influence of BPA.

Many of the physiological effects of BPA have been described in the context of its ability to interact with classic ER (nuclear hormone receptor activity). The estrogenic activity of BPA was reviewed in detail by the NTP-CERHR Expert Panel on Bisphenol A ${ }^{17}$.

In addition to nuclear hormone receptor activity, BPA also impacts cellular physiology through rapid signaling mechanisms to modify the activities of various intracellular signaling networks ${ }^{17}$. The rapid signaling effects of BPA are independent of nuclear hormone receptor activity and occur within minutes or seconds of exposure, initiated by membrane associated or intracellular receptor systems.

- This entero hepatic processs is a slow elimination process of BPA and for the same oral dose, rodents will have a prolonged higher exposure to free, oestrogenically active BPA, compared to primates.

- The oxidation processes of BPA have been in mice-dose administration, suggesting possible formation of metabolites with higher oestrogenic potency.

- Even the importance of first-pass metabolism of BPA is known, the value for human risk assessment of animal studies that have used non-oral routes of administration has 
been questioned. Kinetics also varies markedly with route of administration, dose, age and even gender.

BPA was an androgen receptor antagonist with an IC50 of $2.34 \mu \mathrm{M}$, a concentration that was about 10-fold higher than the EC50 for ER $\alpha$ ago. BPA can also interact with non-classic estrogen receptor systems at similar or lower concentrations than interactions with ER $\alpha$ and $\mathrm{ER} \beta$ mystic activity of $0.272 \mu \mathrm{M}$.

Additionally, the binding affinity of BPA to the ER- $\alpha$ and $\beta$ is approximately 10,000 to100,000 fold lower than that of E2 as judged by three different binding assays across four laboratories. BPA has recently been described as an agonist for the glucocorticoid receptor ${ }^{19}$. Sargis reported that $1 \mu \mathrm{M}$ of BPA (the only concentration tested) could increase luciferase expression in 3T3-L1 pre adipocytes that were transfected with a glucocorticoid response element. Prasanth et al. (2010) used in silico molecular modeling and docking techniques to evaluate BPA's ability to bind to GR.

\subsection{Effect of bpa on intestinal probiotics:}

The origin of probiotics, fermented foods and cultured milk predates recorded history. In 1908, Metchnikoff made observations on human health and longevity that are associated with the ingestion of lactic acid producing bacteria. The concept of $\mathrm{Y}$ probiotics evolved based on such observations. B Probiotics mean B for life and are defined as live microorganisms, which when consumed in adequate amounts.

Probiotics are live microbes that can be formulated into many different types of products, including foods, drugs, and dietary supplements. Species of Lactobacillus and Bifidobacterium are most commonly used as probiotics, but the yeast Saccharomyces cerevisiae and some E. coli and Bacillus species are also used as probiotics.

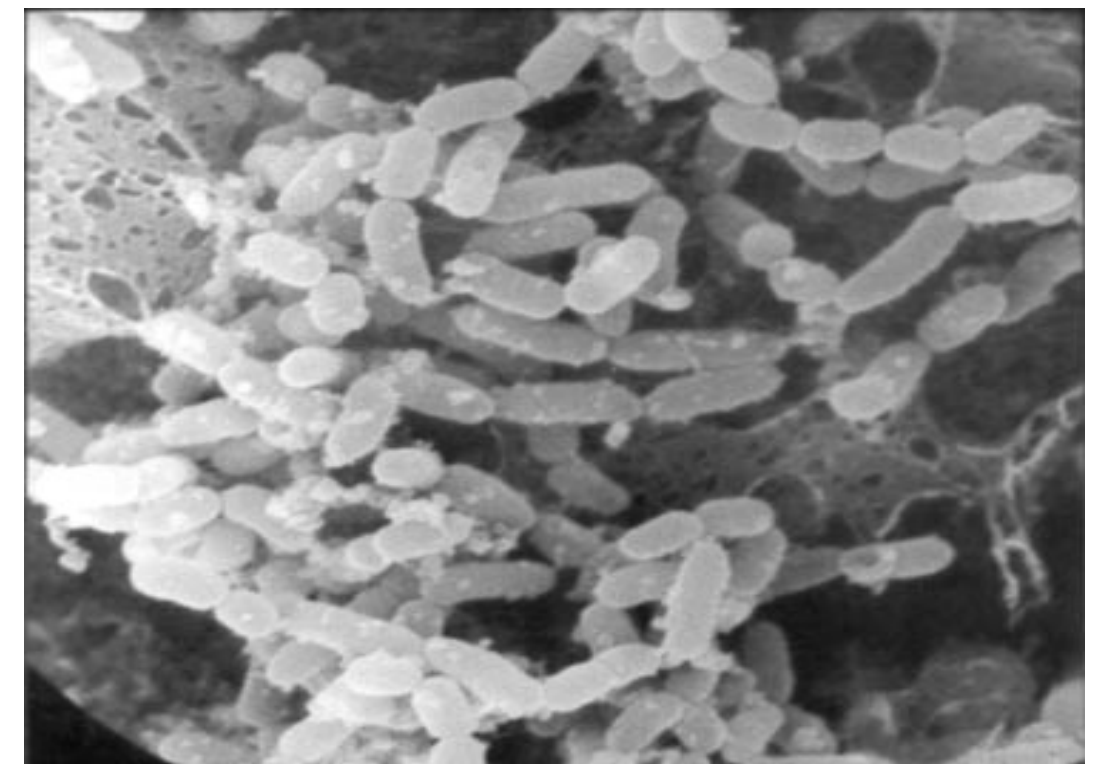

Fig. 6. Electron micrograph of Lactobacillus salivarius 118 adhering to Caco-2 cells.

\section{4. Health claims:}

Probiotics are intended to assist the body's naturally occurring gut microbiota. Studies have documented probiotic effects on a variety of gastrointestinal and extra intestinal disorders, including inflammatory bowel disease (IBD), irritable bowel syndrome (IBS), vaginal infections, and immune enhancement 


\section{5. Mechanisms of action:}

Probiotics affect the intestinal ecosystem by stimulating mucosal immune mechanisms and by stimulating non immune mechanisms through antagonism/competition with potential pathogens. The microbiota, however, participates in the function of the mucosal barrier against the adhesion of pathogenic bacteria, which is at the base of infectious processes. When this barrier function is altered by chemical agents (pesticides,dust etc), antigens, or other stress factors, different types of intestinal disorders can occur, sometimes due to pathogenic bacteria proliferation. Lactic acid bacteria (LAB) are known to produce antibacterial peptides and small proteins called bacteriocins, which enable them to compete against other bacteria in the environment. Lactobacillus salivarius is a promising probiotic candidate frequently isolated from human porcin and avian gastro intestinal tracts (GIT), many of which are producers of unmodified bacteriocins of sub-classes IIa, IIb and IId ${ }^{20}$. It is well characterized bacteriocin producer and probiotic organism ${ }^{21}$. Lsalivarius CECT 5713 fulfills safety criteria for probiotic bacteria ${ }^{22}$ and it has been demonstrated to be safe in animal models.

\section{6. Effect of bpa on different stages of human life:}

A number of effects of BPA in animals have been extensively investigated and target organs identified in repeat-dose animal studies include intestine, liver and kidney. However, the effects of most concern have been those related to the hormonal activity of BPA and potentially related effects on physical, neurological and behavioral development.

The debate about the human health effects of BPA exposure is limited by a lack of epidemiological data. Currently, there is not sufficient statistical power to detect low dose effects or determine all the health consequences of exposure to BPA in humans ${ }^{23}$. Often exposure is considered to be constant over time, with the total dose estimated by the cumulative exposure; it is calculated as the product of concentration anytime. However, if exposure is not constant over time, the same total cumulative exposure delivered in different patterns, may produce different biological effects

\section{7. In fetal stage:}

Now there is concern that BPA is recognized to cross the human placenta, thereby exposing the fetus, the developing fetus is more susceptible than the adult organism to estrogenic chemicals and it has the potential to interfere with the steroid-dependent organization of the neurochemical and neuroendocrine systems, particularly those that are estrogen sensitive. It is in developing brain; alterations in the estrogenic milieu influence various aspects of cellular differentiation, including neuritis extension and branching, synaptic formation, expression of neurotransmitters, cell death and/or survival ${ }^{24}$. Yolton et al., found no association between mothers' urinary BPA concentrations and infant neuro behavior evaluated at 5 weeks of age. These human data, although inconsistent, indicate that prenatal BPA-exposure may affect child behaviors in a sex-dependent manner. However, they are not considered to be adequate ${ }^{25}$.

\section{8. In children:}

Braun et al. (2011) observed associations between mothers' urinary BPA concentrations during pregnancy and anxious, depressive and hyperactive behaviors at 2 (2009) and 3 (2011) years of age, which were more pronounced for girls than for boys. In contrast Perera et al., reported decreased anxious/depressed and aggressive behaviors in 3-5 year old girls but increased aggression and emotionally reactive behaviors in 3-5 year old boys with higher prenatal exposure to BPA (measured in mothers' urine during pregnancy). 


\subsection{In adults:}

Only a few studies have looked at associations between bisphenol A exposure and disorders of reproduction or developmental effects in humans. The studies on human have looked at the relationship between urine or blood concentrations of total or free bisphenol A and a variety of health measures included like:

1. Certain hormones that helps to regulate reproduction markers of DNA damage.

2. Miscarriage,

3. Defects in fetuses,

4. Fertility and obesity in women

5. Effects on the tissue that lines the uterus ("endometrium"),

6. Polycystic ovary syndrome, and birth outcomes and length of gestation.

Several studies in humans report a link between BPA exposure and effects on the reproductive system in adult women, e.g. endometrial hyperplasia ${ }^{27}$, recurrent miscarriages ${ }^{28}$ and polycystic ovary syndrome ${ }^{29,30}$. Hardin (1981) previously reported that intra peritoneal injection of BPA to mated female rats at dose levels of 85 and $125 \mathrm{mg} / \mathrm{kg}$ during gestational days (GD) 1-15 showed decreased number of live fetuses per litter and suppressed fetal body weight and crown-rump length. In addition, the dose of $125 \mathrm{mg} / \mathrm{kg}$ exhibited reduced pregnancy rate and increased incidence of fetal malformations including imperforate anus and enlarged cerebral ventricle.

\section{10. Associations between BPA and reproductive health in women:}

The associations between BPA exposure and breast cancer have been investigated in one case control study in Korean women ${ }^{32}$. BPA levels did not differ between cases and controls in this study. However, major methodological limitations, such as low statistical power, undetectable BPA levels in many of the subjects and cross-sectional design prevents any conclusions to be drawn from this study. No human studies investigating the association between developmental exposure to BPA and effects on the mammary gland were identified.

\section{11. Associations between BPA and obesity in humans:}

A positive relationship between BPA exposure and obesity has been reported in the US general population ${ }^{33}$ and in a small Japanese study in women with and without ovarian dysfunction $^{29}$. Additionally, PCBs are lipophilic and so have the potential to bio accumulate in adipose tissue. This is of particular concern when PCBs accumulate in tissues of pre reproductive or reproductive females, because these compounds can be transferred to the developing offspring either through the placenta or postnatal through lactation.

\section{12. Treatment:}

After the ingestion, a metabolic process is carried out by enzymes primarily in the liver where, the majority of bisphenol A is bound quickly to glucuronic acid to produce bisphenol A-glucuronide. (glucuronidation). As bisphenolA is more soluble in water, it is easier to eliminate in the urine and also minimizes its ability to interact with biological processes in the body.

When rats exposed to BPA in their diet with probiotics, their blood concentrations of BPA dropped dramatically and it was excreted 2.4 times more readily than the non- 
supplemented control group. In other words, probiotics reduced intestinal absorption by boosting BPA excretion and they may suppress BPA's adverse effects on human health.

An easy and inexpensive way to boost the probiotic content of your gut is to take supplements such as IVL's Flora Life - the first and only acid-proof delayed-release probiotic available on the market today. Two capsules a day is all it takes to infuse your digestive tract with 20 billion 'good' bacteria.

Once in your intestines, these delayed release capsules deliver the living probiotics necessary to healthfully balance your gut bacteria and help your body get rid of toxic chemicals much more easily, along with offering multiple other health benefits.( ttp://healthblog.ivlproducts.com/blog/ health-well-being-and-spirituality/probiotics-destroytoxic-chemicals-in-the-gut\#sthash.O5j9OgP. dpuf).

\section{13. Preventive measures:}

\section{13. 1. For infants:}

As research continues, concerned parents can take the following precautionary measures to reduce babies' exposure to BPA:

- Avoiding clear plastic baby bottles or containers with the recycling number 7 and the letters "PC" imprinted on them. Many contain BPA.

- Consider using certified or identified BPA-free plastic bottles.

- Using bottles made of opaque plastic (i.e) made of polyethylene or polypropylene, that do not contain BPA with the recycle symbols 2 or 5 in them.

- Because heat may cause the release of BPA from plastic, consider the following

- Do not heat or boil polycarbonate bottles.

- Do not wash polycarbonate bottles in the dishwasher.

- Glass bottles can be an alternative, but be aware of the risk of injury, if the bottle is dropped or broken.

- Breastfeeding is another way to reduce potential BPA exposure.

- Risks associated with giving infants inappropriate (home-made condensed milk) formulas or alternative (soy or goat) milk is far greater than the potential effects of BPA.

\section{13. 2. For children \&Adults:}

Before getting dental sealants, an individual should check with the dentist about the ingredients in the products they use, as some formulations may leach bisphenol-A

- Using glass, stainless steel, paper, cloth, or ceramic containers for food and beverages.

- Remember that "microwave safe" only means that the container or cling wrap won't

deform; it has nothing to do with your safety.

- Avoiding contact between fatty or acidic foods and plastic

- Recycling of any worn or scratched plastic

- Using ceramic or stainless steel containers.

- Checking the type of plastic in food processor is made from.

- Replace plastic coffee filters with ceramic or metal ones 


\section{13. 3. Handling of BPA:}

- Some cautions are to be followed in handling of BPA in the solid or dust, they include work shoes, gloves, long sleeves and pants or a protective suit such as Tyvek ${ }^{\circledR}$

- When handling phenol, recommended PPE (personal protective equipment) a chemical suit made of non-permeable material equipped with a supplied air respirator.

- Safety glasses side shields or goggles are recommended for eye protection. Gloves that are chemically resistant to BPA should be used.

- Additional PPE is recommended when handling BPA samples in liquid form because, skin exposure to phenol can have dangerous health effects, complete protection is needed when performing this type of task

\section{CONCLUSIONS}

There is substantial evidence indicating that endocrine disruptors to the risk of cancer, developmental problems, diabetes, and possibly also obesity and the metabolic syndrome. Also, it seems highly likely that endocrine disruptors can contribute to infertility and sub fertility. Scientific decisions concerning health risks are generally based on what is known as the "weight-of-evidence." In the case of bisphenol A, evidence from the limited number of studies in humans exposed to bisphenol $\mathrm{A}$ is not sufficient to reach conclusions regarding possible developmental or reproductive hazard. These include studies of traditional designs carried out to assess the toxicity of bisphenol A, as well as a wide variety of studies examining the possibility that exposure to "low" doses of bisphenol A, defined in the NTP Brief on Bisphenol $\mathrm{A}$ as $\leq 5 \mathrm{mg} / \mathrm{kg}$ bw/day during critical periods of development might result in adverse health outcomes later in life due to its estrogenic or other biological properties

A recent guideline study found no evidence that BPA is a developmental neurotoxicant. Concerns are increasing on limiting BPA manufacture use all over the world. But it will take a long time to completely ban the use of BPA in the plastic industries, as industries are trying hard to justify and prove that BPA is not that disastrous, as shown by various studies and researchers all over the world. They have a different point of view, so many more efforts are required to evolve methods for the safe removal of BPA from environment rather than aiming at banning the use of BPA. Internal exposure to free BPA available for biological activity within the body is therefore expected to be very low.

Recent data from measurements of un-conjugated (free) BPA in human blood and urine however suggest higher internal exposure of humans to free BPA. Newborns are expected to be exposed to higher internal BPA values due to immature glucuronidation activity. These discrepancies warrant further investigations for better understanding of toxicokinetics, species and inter-individual differences, possible for other sources of exposure to BPA and potential confounders impacting on the results. An extensive study can even pave the way for the development of probiotics, i.e. live microorganisms which, when administered in adequate amounts, confer a health benefit on the host. These probiotics may be appropriately used for the safe removal of accumulated BPA from living systems. 


\section{References}

[1] Beronius A., Hanberg A., Sources of exposure to bisphenol A. IMM report 2/2011. Institute of Environmental Medicine, Karolinska Institute: ki.se/IMM.

[2] Staples C., Dorn P., Klecka G., O Block S., Harris L A., Chemosphere 36 (1998) 21492173

[3] Biedermann S.1., Tschudin P., Grob K., Analytical and Bioanalytical Chemistry 398 (2010) 571-576.

[4] Welshons W. V., S. C. Nagel, Endocrinology 147(6) (2006) S56-S59.

[5] Shin S.D., Lee B.S., Lee B.M., Han K. C., Kim S.Y., Kwack H.S., Park S.J., Journal of Toxicology and Environmental Health A 64 (2001) 417-426.

[6] Domoradzki J. Y., Pottenger L. H., Thornton C. M., Hansen S. C., Card T.L., Markham D. A., Dryzga, M. D., Shiotsuka, R. N., Waechter J. M., Journal of Toxicological Sciences76 (2003) 21-34.

[7] Pottenger L. H., Domoradzki J. Y., Markham D. A., Hansen S. C., CagenS. Z and

[8] Waechter J. M., Journal of Toxicological Sciences 54 (2000) 3-18.

[9] Pritchett J. J., Kuester R .K., and Sipes I G., Drug Metabolism and Disposition 30 (2002) 1180-1185.

[10] Hugo E.R., Brandebourg T.D., Woo J.G., Loftus J., Alexander J.W., Ben-Jonathan N., Environmental Health Perspective 166 (2008) 1642-1647.

[11] Sohoni P., Sumpter J.P., Journal of Endocrinology 158 (1998) 327-339.

[12] Xu X-H., et al., Hormones and Behavior 58 (2010) 326-333.

[13] Bonefeld-Jorgensen E.C., Hofmeister M.V., Vinggaard A.M., Environ mental Health Prospectus and Supplementation 115 (2007) 69-76.

[14] Smith C.C., Taylor H.S., FASEB Journal 21 (2007) 239-244.

[15] Lee Y.M., et al., Journal of Veterinary Science 8 (2007) 27-38.

[16] Lee L.B., Journal of Neuroscience Research 86 (2008) 2932-2942.

[17] Chung Y.W., Nunez A.A., Clemens L.G., Physiology and Behaviour 74 (2001) 363370 .

[18] Chapin R.E, Adams J., et al., Reproductive Toxicology 83 (2008) 157-395.

[19] Wetherill Y.B., Akingbemi B.T., Reproductive toxicology 24 (2007) 178-198.

[20] Prasanth G.K., Divya L.M., et al., Journal of applied toxicology 30(8) (2010) 769-774.

[21] O’Shea E.F., O’Connor P.M., Raftis E.J., O’Toole P.W., Stanton C., Cotter P.D., Ross R.P., Hill C., Journal of Bacteriology 193 (2001) 6973e-6982e.

[22] Dobson A., Cotter P., Ross P., Hill C., Applied and Environmental Microbiology 78 (2012) 1-6.

[23] Martan R., Jiménez E., Olivares M., Marin M.L., Fernande L., Xaus J., Rodriguez J.M., International Journal of Food Microbiology 112 (2006) 112e-135e. 
[24] Lang, I. A., Galloway T. S., et al., JAMA 300 (2008) 1303-1310.

[25] Yolton K., Xu Y., Strauss D., Altaye M., Calafat A.M., Khoury J., Neurotoxicology and Teratology 33 (2008) 558-566.

[26] Braun J.M., Yolton K., Dietrich K.N., Hornung R., Ye X., Calafat A.M., Lanphear B.P., Environmental Health Perspect 117 (2009) 1945-1952.

[27] Perera F., Tang D.L., Vishnevetsky J., Herbstman J., Calafat A., Xiong W., Rauh V., Wang.S., Environmental Health Perspective 120 (2012) 921-926.

[28] Hiroi H., Tsutsumi O., Takeuchi T., Momoeda M., IkezukiY., Okamura A., Yokota H., Taketani Y., Endocrine Journal 51 (2004) 595-600.

[29] Sugiura-Ogasawara M., Ozaki Y., Sonta S., Makino T., Suzumori K., Human Reproduction 20 (2005) 2325-2329.

[30] Takeuchi T., Tsutsumi O., Ikezuki Y., Takai Y., Taketani Y., Endocrine Journal 51 (2004) 165-169.

[31] Diamanti-Kandarakis E., Bourguignon J.P., Giudice L.C., Endocrine-disrupting 30 (2009) 293-342.

[32] Hardin B..D., Bond G.P., Sikov M.R., Andrew F.D., Beliles R.P., Niemeier R.W., Scandinavian Journal of Work Environment \& Health 7 (1981) 66-75.

[33] Yang M., Ryu JH., Jeon R., Kang D., Yoo KY., Archives of Toxicology 83 (2009) 281285.

[34] Carwile J.L., Michels K.B., Environmental Research 111 (2006) 825-830. 